\title{
Excesso de peso e obesidade em escolares: associação com fatores biopsicológicos, socioeconômicos e comportamentais
}

\author{
Overweight and obesity in school children: association between \\ biopsychological, socioeconomic and behavioral factors
}

1 Departamento de Educação Física, Centro de Ciências da Saúde e do Esporte, Universidade do Estado de Santa Catarina (Udesc), Florianópolis, SC,

Brasil; Faculdade de Motricidade Humana, Universidade

Técnica de Lisboa, Portuga

${ }^{2}$ Centro de Ciências da

Saúde e do Esporte, Udesc,

Florianópolis, SC, Brasil

${ }^{3}$ Departamento de Educação

Física e Fisioterapia, Faculdade

Porto das Águas (FAPAG) e

Instituto de Ensino Superior

(IES) da Grande Florianópolis:

Universidade Federal de Santa

Catarina (UFSC), Programa de

Pós-Graduação em Atividade Física

e Saúde, Florianópolis, SC, Brasil

${ }^{4}$ Serviço Social da Indústria

(SESI) e UFSC, Programa de Pós-

Graduação em Atividade Física e

Saúde, Florianópolis, SC, Brasil

${ }^{5}$ Departamento de Educação Física

Centro de Ciências da Saúde e

do Esporte, Udesc, Florianópolis,

SC, Brasil; Faculdade de

Motricidade Humana, Universidade

Técnica de Lisboa, Portugal

${ }^{6}$ Departamento de Ciências

da Saúde, Centro de Ciências

da Saúde e do Esporte, Udesc,

Florianópolis, SC, Brasil

Correspondência para:

Adriana Coutinho de

Azevedo Guimarães

Rua Bias Peixoto, 187, ap. 201,

88085-480 - Florianópolis,

SC, Brasil

nanaguim@terra.com.br

Recebido em 8/Jun/2011

Aceito em 17/Fev/2012
Adriana Coutinho de Azevedo Guimarães ', Isabela Feijó², Amanda Soares ${ }^{3}$, Sabrina Fernandes ${ }^{4}$, Zenite Machado ${ }^{5}$, Sílvia Rosane Parcias ${ }^{6}$

\begin{abstract}
RESUMO
Objetivo: Verificar a associação dos fatores biopsicológicos, socioeconômicos e comportamentais em escolares com excesso de peso e obesidade. Sujeitos e métodos: A amostra foi constituída por 393 escolares da rede pública e privada de Florianópolis/SC, com idade 9,9 \pm 1,7 anos, sendo $41 \%$ do sexo masculino. Foi aplicado um questionário adaptado de Oliveira e cols. em forma de entrevista e realizada a avaliação antropométrica (massa corporal e estatura) para o cálculo do índice de massa corporal (IMC) e classificação de excesso de peso e obesidade conforme Conde e Monteiro. Resultados: Em relação à distribuição de obesidade e excesso de peso por sexo, verificou-se uma distribuição equitativa entre as meninas, enquanto nos meninos o excesso de peso representou $77 \%$ da amostra. Houve associações entre sexo feminino, estrato econômico, etnia, escola e qualidade dietética, com valores de $\mathrm{p}$ variando de $<0,001 \mathrm{a}$ 0,003. Conclusão: Os fatores biopsicológicos, socioeconômicos e comportamentais parecem interferir no excesso de peso e na obesidade em escolares de Florianópolis. Arq Bras Endocrinol Metab. 2012;56(2):142-8
\end{abstract}

\section{Descritores}

Obesidade; atividade motora; excesso de peso; escolares; hábitos alimentares

\begin{abstract}
Objective: To verify the association between the behavioral, biopsychological, and socioeconomic factors in overweight and obese students. Subjects and methods: The sample involved 393 students from state and private schools in Florianópolis/SC, mean age of $9.9 \pm 1.7$, with $41 \%$ male subjects. A questionnaire adapted from Oliveira e cols. was applied in the interview; anthropometric information (body mass and height) was used to calculate the BMI, and individuals were classified in overweight and obese according to Conde and Monteiro. Results: As for the distribution of obesity and overweight by gender, girls showed a similar degree, while among boys, overweight individuals represented $77 \%$ of the sample. There were associations between female gender, economic status, ethnicity, school, and type of food with $p$ ranging from $<0.001$ to 0.003 . Conclusion: It was found that the behavioral, biopsychological, and socioeconomic factors seem to interfere with overweight and obesity in students of Florianópolis. Arq Bras Endocrinol Metab. 2012;56(2):142-8
\end{abstract}

\section{Keywords}

Obesity; motor activity; overweight; students; nutritional habits

\section{INTRODUÇ̃̃O}

$\mathrm{O}$ excesso de peso e a obesidade são caracterizados por acúmulo de gordura corporal que representa risco para a saúde (1). A prevalência da obesidade é um problema de saúde pública com caráter epidêmico (2), sendo por vezes classificada como pandemia (3) devido 
ao crescimento nas últimas décadas, principalmente na população infantil e nos países em desenvolvimento (4).

Dados da Organização Pan-Americana da Saúde (OPAS) (5) e da Organização Mundial da Saúde (OMS) (6) sustentam que a prevalência de obesidade infantil tem aumentado em torno de $10 \%$ a $40 \%$ na maioria dos países europeus nos últimos 10 anos, e no Brasil o índice de obesidade infanto-juvenil subiu 240\% nas últimas duas décadas (5). Em Florianópolis, a prevalência de excesso de peso e obesidade em escolares de 7 a 9 anos de uma escola pública foi de $17 \%$ e 7,0\%, respectivamente (7).

Apesar de se manifestar em qualquer época da vida, existem dois picos importantes na infância nos quais ocorre aumento de células gordurosas e gordura corporal favorecido pelo crescimento desacelerado (8). Esses picos ocorrem no primeiro ano de vida e entre os 5 e 7 anos de idade. Sabe-se que quanto mais intenso e precoce o aumento da gordura corporal, maior o risco de persistência da obesidade ao longo da vida (9). Estudos demonstram que aos 6 meses, 4 anos (10) e 5 anos (11) de idade $50 \%, 20 \%$ e $80 \%$ das crianças respectivamente que estão obesas permanecerão como tal na fase adulta, tornando-se potenciais portadoras de doenças cardiovasculares, hipertensão arterial sistêmica, dislipidemia aterogênica, apneia do sono, aterosclerose precoce, diabetes e doença hepática gordurosa não alcoólica (12).

Os principais fatores do desenvolvimento da obesidade parecem ser os biopsicológicos $(8,13)$, socioeconômicos (14) e comportamentais $(8,11,15)$. Neste sentido, o presente estudo teve como objetivo verificar a associação desses três fatores com o excesso de peso e a obesidade em escolares, visto que esse conhecimento é essencial para o planejamento de ações preventivas e de reabilitação na área de educação e saúde.

\section{SUJEITOS E MÉTODOS}

O Comitê de Ética em Pesquisa com Seres Humanos (CEPSH) da Udesc aprovou o estudo com número de protocolo 79/2009. A autorização dos pais ou responsáveis foi obtida por meio da assinatura do termo de consentimento livre e esclarecido.

O estudo, de corte transversal, foi realizado no período de novembro de 2009 a março de 2010 , em três escolas (2 públicas e 1 privada) de Florianópolis, SC, Brasil. Os alunos matriculados no ensino fundamental, nas turmas de $1^{a}$ a $6^{a}$ série, compuseram essa população, perfazendo um total de 3.264 indivíduos. Após o cálculo amostral para representatividade, chegou-se ao número mínimo aceitável de 344 escolares com erro de $5 \%$.

Os alunos foram selecionados de forma aleatória respeitando-se o critério de inclusão adotado, ao qual o índice de massa corporal (IMC) deveria indicar excesso de peso ou obesidade, conforme a classificação de Conde e Monteiro (16). Assim, a amostra foi composta por 393 escolares, com 9,9 $\pm 1,7$ anos de idade, sendo $41 \%$ do sexo masculino, $61 \%$ com excesso de peso e $39 \%$ obesos.

Para responder aos objetivos do estudo, foi utilizado um questionário adaptado de Oliveira e cols. (8), o qual foi submetido à validação de face com índice de validade de 0,92 e índice de clareza de 0,99 . O questionário continha cinco partes: a) fatores biológicos: sexo, faixa etária, etnia; b) avaliação antropométrica (massa corporal e estatura); c) fatores comportamentais: hábitos alimentares, atividade física e lazer; d) fatores psicológicos: dinâmica familiar, repetência escolar, série e imagem pessoal; e) fatores socioeconômicos dos pais ou responsáveis dos escolares (ABEP) (17). As questões socioeconômicas foram respondidas pelos pais e as demais foram questionadas aos alunos por meio de entrevista, durante as aulas de Educação Física escolar.

Para a avaliação antropométrica, foi utilizada uma balança digital da marca Plenna, modelo MEA 08-128, com capacidade de até $150 \mathrm{~kg}$ para aferir a massa corporal e uma fita métrica da marca Lufkin - $3 \mathrm{~m}$ ultralok para medir a estatura. Posteriormente, foi calculado o IMC $\mathrm{kg} / \mathrm{m}^{2}$ (constitui-se do peso em $\mathrm{kg}$ dividido pelo quadrado da estatura em metro).

No tratamento estatístico, utilizou-se análise descritiva (frequência simples, percentual, média e desvio-padrão) e inferencial (teste do qui-quadrado). Adotou-se o nível de significância de $95 \%$, utilizando-se o pacote estatístico SPSS versão - 17.0. Os resultados são apresentados no formato de tabelas. Como algumas crianças não responderam a todas as questões, alguns indicadores apresentam " $\mathrm{n}$ " inferior ao total.

\section{RESULTADOS}

Com o intuito de analisar as relações entre os fatores socioeconômicos, biopsicológicos e comportamentais entre os dois grupos - excesso de peso e obesidade -, os resultados são apresentados por vezes considerando a totalidade da amostra e outrora discriminados pelo excesso de peso e obesidade. 


\section{Fatores biológicos e socioeconômicos}

Sobre o fator econômico verificou-se que $29 \%$ dos pais pertenciam ao estrato A e $52 \%$, ao B. A grande maioria ( $77 \%$ das mães e $93 \%$ dos pais) exercia atividade remunerada, sendo predominante a atividade no setor 2 (empresa e instituições privadas, comércio, autônomo) e $67 \%$ se consideravam de etnia branca. Em relação à distribuição de obesidade e excesso de peso por sexo, verificou-se uma distribuição equitativa entre as meninas, enquanto nos meninos o excesso de peso representou $77 \%$ da amostra.

$\mathrm{Na}$ tabela $\mathrm{l}$ é apresentada a associação entre os fatores biológicos e o IMC. Foi observada diferença significativa entre os sexos $(\mathrm{p}<0,001)$ indicando valores mais elevados de IMC para as meninas. $\mathrm{O}$ resultado relativo à etnia $(\mathrm{p}=0,003)$ sugere que a obesidade é mais frequente entre crianças que se denominaram mulatas e negras. Quanto ao fator socioeconômico, verificou-se que a classe social se associa à ocorrência de excesso de peso/ obesidade $(\mathrm{p}<0,001)$, com valores crescentes na medida em que diminui o poder aquisitivo. Paralelamente foi observado que os casos de obesidade são menos frequentes em escolares da rede privada de ensino e maiores entre os escolares da rede municipal $(\mathrm{p}=0,001)$.

\section{Fatores psicológicos}

Considerando os fatores psicológicos, o índice de repetência escolar foi de $15 \%$, sendo que $4,0 \%$ repetiram mais de uma vez. A repetência escolar ocorreu de forma mais significativa entre os alunos com excesso de peso $(\mathrm{p}=0,05)$.
Verificou-se também a realização de dieta entre os investigados. Nesta variável, 38\% dos alunos fazem dieta, sendo que o mesmo percentual relatou não fazer, pois acredita estar no peso ideal. Os demais $(24 \%)$ não fazem, embora tenham relatado que precisam fazer. $\mathrm{Na}$ análise intergrupos, respaldada pelo teste qui-quadrado $(\mathrm{p}<0,001)$, verifica-se que os escolares com excesso de peso e obesos comportam-se diferentemente em relação à adoção de uma dieta. No subgrupo com excesso de peso, existe certo equilíbrio entre os que realizam dieta $(33 \%)$, os que não realizam por julgarem que o peso está adequado (34\%) e os que não realizam, mas consideram que deveriam (33\%). Já no subgrupo dos obesos $46 \%$ afirmam categoricamente realizar, $45 \%$ não realizam, pois estão contentes com seu peso, sendo que apenas $9,0 \%$ não se submetem à dieta embora a julgue necessária.

Ainda seguindo pelos fatores psicológicos, observou-se na tabela 2 que os escolares apresentaram uma imagem pessoal positiva na medida em que $47 \%$ deles percebem-se como crianças nos padrões normais de massa corporal, independentemente de estarem com excesso de peso ou obesos e apenas $28 \%$ se acham fora desses padrões (como referido: "gordos"). Esse resultado corrobora com o percentual $(21 \%)$ daqueles que sequer possuem uma opinião a respeito, o que pode denotar uma despreocupação sobre o assunto. Apesar de $48 \%$ acharem sua aparência boa, $63 \%$ gostariam de mudar seu corpo, e mais da metade dos escolares (81\%) está insatisfeita com o volume abdominal.

Tabela 1. Associação entre fatores biológicos, socioeconômico e IMC

\begin{tabular}{|c|c|c|c|c|c|c|}
\hline Variáveis & $\mathbf{n}$ & Excesso de peso & & Obesidade & & Valor de $p$ \\
\hline Sexo & 393 & & $\%$ & & $\%$ & $<0,001$ \\
\hline Masculino & & 123 & 76,9 & 37 & 23,1 & \\
\hline Feminino & & 118 & 50,6 & 115 & 49,4 & \\
\hline Socioeconômico & 393 & & & & & $<0,001$ \\
\hline Classe A & & 92 & 80,7 & 22 & 19,3 & \\
\hline Classe B & & 114 & 55,9 & 90 & 44,1 & \\
\hline Classe C e D & & 35 & 46,7 & 40 & 53,3 & \\
\hline Etnia & 392 & & & & & 0,003 \\
\hline Branco & & 172 & 65,2 & 92 & 34,8 & \\
\hline Mulato & & 55 & 59,8 & 37 & 40,2 & \\
\hline Negro & & 13 & 36,1 & 23 & 63,9 & \\
\hline Escola & 393 & & & & & $<0,001$ \\
\hline Pública municipal & & 18 & 34,0 & 35 & 66,0 & \\
\hline Pública estadual & & 101 & 52,1 & 93 & 47,9 & \\
\hline Privada & & 122 & 83,6 & 24 & 16,4 & \\
\hline
\end{tabular}

IMC: índice de massa corporal. 
Tabela 2. Fatores psicológicos: imagem pessoal dos escolares matriculados em escolas públicas e privada de Florianópolis

\begin{tabular}{lccc}
\hline Variáveis & $\mathbf{n}$ & $\mathbf{F}$ & $\mathbf{\%}$ \\
\hline Percepção da imagem & 393 & & \\
Magro & & 16 & 4,1 \\
Normal & & 110 & 46,8 \\
Gordo & & 83 & 21,1 \\
Não sabe & 393 & & \\
Aparência & & 188 & 47,9 \\
Boa & & 89 & 22,6 \\
Média & & 29 & 7,4 \\
Ruim & & 87 & 22,1 \\
Não pensa nisso & & \\
Mudança corpo & 393 & 247 & 62,8 \\
Sim & & 146 & 37,2 \\
Não & & & \\
Mudança parte do corpo & 247 & 3 & 1,3 \\
Braço & & 19 & 7,6 \\
Rosto & & 2,8 \\
Perna & & 200 & 81,0 \\
Barriga & 16 & 0,8 \\
Bumbum & & \\
Outra parte & & & \\
\hline
\end{tabular}

F: frequência.

\section{Fatores comportamentais}

Os fatores comportamentais relacionados à atividade física, presentes na tabela 3 , não apresentaram associação significativa. No entanto, podemos observar a grande participação dos escolares em atividades esportivas ou pré-esportivas nos dias úteis.

Em relação à prática de atividade física, $98 \%$ dos escolares relataram participar das aulas de Educação Física curricular, tendo gosto por estas (95\%), não havendo diferenças percentuais entre os grupos. Além da Educação Física curricular, $57 \%$ realizavam outras práticas de atividade física durante a semana, $30 \%$ duas vezes por semana e $16 \%$ apenas no fim de semana. A ativida- de física mais procurada diferiu entre os grupos, sendo para os obesos a dança $(26 \%)$ e a natação (18\%) e, para os com excesso de peso, o futebol $(24 \%)$ e a natação (17\%). Observaram-se escores bem diferentes para a modalidade dança nos grupos, sendo realizada por $13 \%$ daqueles com excesso de peso e $26 \%$ dos obesos.

Quanto ao lazer, verificou-se que as atividades esportivas ou pré-esportivas foram as mais citadas quando questionados sobre o que gostam de fazer para se divertir $(66 \%)$, sua brincadeira predileta $(59 \%)$ e do que geralmente brincam $(57 \%)$. A rua foi o local mais citado para brincar $(29 \%)$, seguido do quintal de casa $(28 \%)$ e do interior de casa $(27 \%)$.

Com relação à atividade passiva, 99\% dos escolares assistem à TV, dos quais $41 \%$ o fazem de 2 a 3 horas por dia durante a semana e $38 \%$ assistem 4 horas ou mais aos fins de semana. Quanto ao uso do computador, $86 \%$ o utilizam, sendo $40 \% \mathrm{l}$ hora por dia durante a semana, e $28 \%$ de 2 a 3 horas por dia aos fins de semana. O videogame é jogado por $73 \%$ dos escolares, dos quais $41 \%$ passam 1 hora por dia durante a semana e $30 \% 1$ hora por dia aos fins de semana ao aparelho. Dos escolares que frequentam o shopping, $55 \%$ vão para se divertir e apenas $8 \%$, para se alimentar.

Na tabela 4, são apresentados os resultados referentes aos fatores comportamentais - hábitos alimentares. De modo geral, verificou-se de acordo com os relatos que $93 \%$ dos escolares tomam refrigerantes e $87 \%$ suco natural, $92 \%$ alimentam-se de frutas e $75 \%$ de verduras, e $57 \%$ também ingerem fast food, doces e guloseimas. Quanto à escolha da refeição, o almoço/jantar foi o preferido por $51 \%$ dos escolares.

Analisando os resultados dos dois grupos discriminados, os lanches/fast food foram preferência alimentar dos escolares que apresentam excesso de peso. Porém, aqueles que apresentam obesidade preferem frutas, sendo que a qualidade dietética associou-se à ocorrência de excesso

Tabela 3. Associação entre IMC e fatores comportamentais - AF

\begin{tabular}{|c|c|c|c|c|c|c|}
\hline Variáveis & $\mathbf{n}$ & Excesso de peso & & Obesidade & & Valor $p$ \\
\hline Educação Física Escolar & 393 & & $\%$ & & $\%$ & 0,719 \\
\hline $\operatorname{Sim}$ & & 236 & 61,5 & 148 & 38,5 & \\
\hline Não & & 5 & 55,6 & 4 & 44,4 & \\
\hline AF semana & 393 & & & & & 0,121 \\
\hline $\operatorname{Sim}$ & & 146 & 64,6 & 80 & 35,4 & \\
\hline Não & & 95 & 56,9 & 72 & 43,1 & \\
\hline AF fim de semana & 393 & & & & & 0,504 \\
\hline Sim & & 41 & 65,1 & 22 & 34,9 & \\
\hline Não & & 200 & 60,6 & 130 & 39,4 & \\
\hline
\end{tabular}

IMC: índice de massa corporal; AF: atividade física. 
Tabela 4. Associação entre IMC e fatores comportamentais - Hábitos alimentares

\begin{tabular}{|c|c|c|c|c|c|c|}
\hline Variáveis & $\mathbf{n}$ & Excesso de peso & & esidad & & Valor de $p$ \\
\hline Qualidade dietética & 390 & & $\%$ & & $\mathrm{~N}$ & 0,001 \\
\hline Frutas & & 36 & 15,1 & 45 & 29,8 & \\
\hline Verduras & & 6 & 2,4 & 8 & 5,2 & \\
\hline Lanches/Fast food & & 101 & 42,3 & 43 & 28,5 & \\
\hline Doces/Guloseimas & & 47 & 19,7 & 33 & 21,9 & \\
\hline Leite/Carne/Ovos & & 49 & 20,5 & 22 & 14,6 & \\
\hline Refeição & 384 & & & & & 0,130 \\
\hline Café da manhã & & 42 & 18,0 & 39 & 26,0 & \\
\hline Lanches & & 63 & 26,9 & 41 & 27,3 & \\
\hline Almocco/Jantar & & 129 & 55,1 & 70 & 46,7 & \\
\hline
\end{tabular}

IMC: índice de massa corporal.

de peso $(\mathrm{p}=0,001)$. Considerando a refeição preferida, não foi observada diferença significativa entre estas ( $\mathrm{p}$ $=0,130)$ por meio da aplicação do teste qui-quadrado.

Os escolares que fazem dieta tendem a privilegiar mais o café da manhã quando comparados com os que não fazem porque o peso está bom $(\mathrm{p}=0,024)$ e com os que não fazem mas precisam $(\mathrm{p}=0,025)$. O grupo que faz dieta também janta mais frequentemente do que os escolares que precisam fazer dieta, mas não o fazem $(\mathrm{p}=0,001)$.

\section{DISCUSSÃO}

Tendo como principal objetivo deste estudo verificar a associação entre os fatores biopsicológicos, socioeconômicos e comportamentais em escolares com excesso de peso e obesidade, encontram-se resultados que apóiam $(18,19)$ os obtidos, pois relataram que a obesidade é significativamente mais prevalente entre o sexo feminino, fato que seria justificado porque, em média, os meninos têm um gasto calórico mais elevado. Já o Consenso Latino-Americano (13) de obesidade relata que na infância a ocorrência da obesidade não difere entre os sexos.

Assim, este estudo ainda apresentou escolares que se declaravam de etnia branca mais propensa ao excesso de peso e os negros à obesidade. Esses resultados também encontram similares na literatura, tais como o de Oliveira e cols. (8). Quando se relaciona ao fator psicológico, mais diretamente à imagem pessoal, Carvalho e cols. (20) apresentam resultados semelhantes de uma imagem pessoal positiva, verificando que, embora o autoconceito de crianças obesas não tenha sido totalmente negativo, questões diretamente relacionadas com a gordura corporal obtiveram, em sua maioria, respostas afirmativas. $\mathrm{O}$ acesso ao autojulgamento facilita a atuação dos profissionais e a aceitação por parte da criança em relação à sua condição, possibilitando a intervenção e a mudança do quadro nutricional desta.
No entanto, o presente estudo revelou que a atuação de profissionais e a consequente adoção de medidas para o controle do avanço dessa pandemia podem ser prejudicadas, pois aproximadamente um terço da amostra está satisfeita com o excesso de peso ou obesidade. Esse aspecto é preocupante na medida em que o excesso de peso ou a obesidade são padrões que tendem a persistir ao longo da vida (9).

Os resultados desse estudo apresentam fraca associação entre repetência escolar e obesidade, embora tal associação tenha sido observada em relação ao excesso de peso. Outros estudos sustentam que as chances de repetência escolar parecem aumentar à medida que o percentil diminui, mostrando uma associação inversa entre as variáveis $(8,21)$. Investigou-se também uma possível influência da origem escolar na prevalência de sobrepeso e obesidade. Considerando as redes de ensino privado, público municipal e estadual, observou-se diferença significativa. A obesidade é mais frequente entre os escolares da rede pública municipal e menos entre aqueles da rede privada. Por outro lado, a rede privada congrega o maior número com sobrepeso, resultados esses que concordam parcialmente com o estudo de Suñé e cols. (22), pois estes observaram que alunos matriculados em escolas estaduais apresentam um risco $33 \%$ menor de desenvolver obesidade que escolares da rede de ensino municipal, e alunos de escolas privadas apresentam um risco $53 \%$ maior para o desenvolvimento da obesidade.

Em relação ao fator socioeconômico, o excesso de peso e a obesidade estão associados de forma significativa aos estratos economicamente favorecidos de acordo com Silva e cols. (23), sendo que no presente estudo os resultados apresentam os escolares nessa condição fazendo parte do estrato econômico B. Esse fato pode estar relacionado ao acesso facilitado aos alimentos com elevada densidade energética e à tecnologia que facilita a 
ocupação do tempo de lazer com atividades sedentárias (23). Nessa relação, observam-se o comprometimento da saúde e o aumento do risco para o desenvolvimento de disfunções metabólicas em idades precoces (24).

Considerando os fatores comportamentais relacionados aos hábitos alimentares, os resultados não apresentaram associação entre refeição e IMC, o que são semelhantes aos de Fagundes e cols. (25). A preferência é pelo almoço e jantar por parte dos escolares com excesso de peso e obesidade. Os autores acrescentam ainda que a preferência pelo café da manhã pode acontecer por parte dos eutróficos e desnutridos. $\mathrm{O}$ fato de crianças com sobrepeso/obesidade não realizarem o desjejum pode acarretar em um aumento significativo do peso quando comparado aos indivíduos que fazem o desjejum diariamente (26).

Neste estudo, o fast food aparece como preferência alimentar das crianças de vários estratos econômicos. Considera-se esse resultado negativo levando em conta os atuais elevados índices de excesso de peso e obesidade, sendo que sua diminuição reduziria a ingestão calórica (27). Variáveis relacionadas aos fatores comportamentais, como a dieta, influenciam diretamente os padrões alimentares, como migração interna, alimentação fora de casa, crescimento na oferta de refeições rápidas (fast food) e ampliação do uso de alimentos industrializados (2). Em decorrência dessas, o consumo de alimentos altamente calóricos eleva em mais de $50 \%$ o risco de apresentar excesso de peso/obesidade em escolares, confirmando a associação estatisticamente significante (22) entre alimentos do tipo lanches/fast food, doces/guloseimas e a ocorrência de obesidade.

A alimentação hipercalórica (2) (por meio do consumo excessivo de açúcares simples, de gorduras animais, de ácidos graxos saturados, de gorduras trans) aliada ao sedentarismo crescente $(8)$ e outras práticas não saudáveis contribui para esse panorama. A prática alimentar e o nível de conhecimento em nutrição estão associados com a obesidade. $\mathrm{O}$ conhecimento restrito e hábitos alimentares menos saudáveis aumentam em cinco vezes as chances do desenvolvimento da obesidade (28). A preferência alimentar das crianças é influenciada diretamente pela cultura e principalmente pelos hábitos dos pais (8), além do fator socioeconômico (14). Um estudo verificou que $52 \%$ das crianças filhas de pai e mãe obesos desenvolveram a obesidade, $50 \%$ quando apenas um dos pais apresentava o caso clínico e $20 \%$ quando nenhum dos pais apresentava acúmulo excessivo de gordura corporal (15). Em outro estudo, as estimativas são mais elevadas. A probabilidade para criança com pais obesos desenvolver obesidade é de $80 \%$. Há uma redução para $40 \%$ quando apenas o pai ou a mãe apresenta esse quadro (11).

Como explicitado anteriormente, a alimentação isoladamente não é fator determinante para o expressivo aumento dos índices de excesso de peso/obesidade. Entre as inúmeras variáveis que contribuem com o desenvolvimento desse quadro clínico, destaca-se a falta de atividade física regular (24). Contrariando os resultados encontrados nos fatores comportamentais direcionados à atividade física, Costa (29) observou forte associação entre quantidade de sessões semanais de atividade física ou Educação Física sistematizada e o IMC, percebendo a existência de uma relação inversa entre as variáveis. Os escolares da pesquisa que participaram ativamente das aulas de Educação Física, pelo menos três vezes por semana, não foram classificados como obesos. Sugere-se que a falta de associação entre IMC e atividade física encontrada aqui se pode referir à quantidade insuficiente de sessões semanais destinadas à prática regular de atividade física. Segundo a OMS (6), a promoção e a manutenção da saúde exigem os níveis mínimos de atividade física, para crianças e jovens ( 5 a 18 anos), de 60 minutos de atividade moderada a vigorosa por dia. Pouca prática moderada e nenhuma vigorosa (18) é resultado da substituição de atividades que necessitam um maior gasto energético por atividades com caráter sedentário (30).

O estudo de Baruk e cols. (18) apresenta considerações sobre as atividades passivas e relata que estas aparecem como importante causa do aumento do IMC, podendo mostrar correlação positiva com a obesidade. O tempo dedicado à permanência em frente à TV, videogame e computador mostrou-se superior ao destinado à prática de atividade física, porém a diferença entre eutróficos e obesos não é a ausência, mas a duração da prática sedentária. A permanência por mais de 4 horas e $30 \mathrm{mi}-$ nutos em atividades sedentárias são variáveis comportamentais que corroboram para a prevalência de excesso de peso e obesidade (22), observação também referenciada neste estudo. Existe uma tendência das crianças em ocuparem seu tempo com atividades que necessitem um menor gasto calórico, favorecendo um balanço energético positivo que tende ao acúmulo de gordura corporal. Somado a isso, a vontade de comer estimulada pelo ato de assistir à TV são variáveis que influenciam diretamente à ocorrência de obesidade na infância (30). O hábito de assistir à TV por mais de três horas, além de estar associado ao excesso de peso, relaciona-se também à baixa aptidão física e à dislipidemia na vida adulta (4). Crianças eutróficas passam menos tempo assistindo à TV e jogando videogame que crianças com excesso de peso $(4,30)$. 
Considerando que crianças mais ativas apresentam índice de massa corporal e percentual de gordura menos elevado, percebe-se que a atividade física é fator protetor contra o excesso de peso e obesidade (18).

Após discorrer e discutir com diversos autores, pode-se observar que a principal limitação deste estudo baseia-se no uso de questionário como instrumento, uma vez que se trata de uma metodologia associada a um erro de 35\%-50\% (31) de subestimação. Além disso, os escolares em muitos momentos se contradizem nas respostas, dificultando a análise dos dados.

No entanto, destacam-se a quantidade e a intensidade insuficiente da prática de atividade física por parte dos escolares, favorecendo um balanço energético positivo devido à maior ingestão que gasto calórico. Além disso, os hábitos e as preferências alimentares não saudáveis fortalecem esse quadro, nos quais os estratos mais elevados economicamente favorecem o acúmulo excessivo de gordura corporal em função do acesso facilitado a alimentos mais energéticos e à tecnologia, contribuindo com o aumento do tempo destinado às atividades sedentárias. Sendo assim, sugerem-se estudos longitudinais que investiguem os fatores biopsicológicos, socioeconômicos e comportamentais relacionados à obesidade em longo prazo, com maior atenção na quantidade e na intensidade da atividade física e alimentação diária.

Declaração: os autores declaram não haver conflitos de interesse científico neste estudo.

\section{REFERÊNCIAS}

1. World Health Organization. Health topics: obesity. Disponivel em: <http://www.who.int/topics/obesity/en/>. Acesso em: Agos 12, 2009.

2. Mendonça CP, Anjos LA. Aspectos das práticas alimentares e da atividade física como determinantes do crescimento do excesso de peso/obesidade no Brasil. Cad Saúde Pública. 2004;20:698-709.

3. Troncon JK, Gomes JP, Guerra-Júnior G, Lalli CA. Prevalência de obesidade em crianças de uma escola pública de um ambulatório geral de Pediatria de hospital universitário. Rev Paul Pediatria. 2007;25:305-10.

4. Nunes MMA, Figueiroa JN, Alves JGB. Excesso de peso, atividade física e hábitos alimentares entre adolescentes de diferentes classes econômicas em Campina Grande (PB). Rev Assoc Med Bras. 2007;53;130-4.

5. Organização Pan-Americana de Saúde/Organização Mundial da Saúde. Doenças crônico-degenerativas e obesidade: estratégia mundial sobre alimentação saudável, atividade física e saúde. Brasília; 2003.

6. World Health Organization. Benefits of Physical Activity. Disponível em: http://www.who.int/dietphysicalactivity/factsheet_benefits/en/index.html. Acesso em: Abr 25, 2010.

7. Soa C, Vasconcelos FAG, Assis MAA, Grosseman S, Luna MEP. Prevalência de excesso de peso e obesidade em escolares de uma escola pública de Florianópolis, Santa Catarina. Rev Bras Saúde Matern Infant. 2004;4:391-7.

8. Oliveira AMA, Cerqueira EMM, Souza JS, Oliveira AC. Excesso de peso e obesidade infantil: influência de fatores biológicos e ambientais em Feira de Santana, BA. Arq Bras Endocrinol Metab. 2003;47:144-50.
9. Boa-Sorte N, Neri LA, Leite MEQ, Brito SM, Meirelles AR, Luduvice FBS, et al. Percepção materna e autopercepção do estado nutricional de crianças e adolescentes de escolas privadas. J Pediatr. 2007;83:349-56.

10. Sichieri R, Souza RA. Estratégias para prevenção da obesidade em crianças e adolescentes. Cad Saúde Pública. 2008;24 supl 2:s209-23.

11. Ramos AMPP, Barros Filho AA. Prevalência da obesidade em adolescentes de Bragança Paulista e sua relação com a obesidade dos pais. Arq Bras Endocrinol Metab. 2003;47:663-8.

12. Kong AP, Chow CC. Medical consequences of childhood obesity: a Hong Kong perspective. Res Sports Med. 2010;18:16-25.

13. Consenso Latino-Americano de Obesidade. Disponível em: <http:// www.abeso.org.br/pdf/consenso.pdf>. Acesso em: Abr 20, 2010.

14. Campos LA, Leite AJM, Almeida PC. Nível socioeconômico e sua influência sobre a prevalência de excesso de peso e obesidade em escolares adolescentes do município de Fortaleza. Rev Nutr. 2006;19:531-8.

15. Simon VGN, Souza JMP, Souza SB. Aleitamento materno, alimentação complementar, excesso de peso e obesidade em pré-escolares. Rev Saúde Pública. 2009;43:60-9.

16. Conde WL, Monteiro CA. Brazilian body mass index cut off points for evaluation of nutritional status from children and adolescents. J Pediatr. 2006;82:266-72.

17. ABEP. Critério Padrão de Classificação Econômica Brasil/2008. Disponível em: <http://www.viverbem.fmb.unesp.br/docs/classificacaobrasil.pdf>. Acesso em: Out 23, 2008.

18. Baruki SBS, Rosado LEFPL, Rosado GP, Ribeiro RCL. Associação entre estado nutricional e atividade física em escolares da Rede Municipal de ensino em Corumbá - MS. Rev Bras Med Esporte. 2006;12:90-4.

19. Romero A, Lucca A, Soares EB, Manoel HV Filho, Aldenir OR Jr, Barbosa A. Prevalência de excesso de peso e obesidade em crianças de 6 a 10 anos de idade, matriculadas em escolas públicas de Guarulhos/SP. Edição Espacial da Rev Bras Ciên Mov. 2009;17:137.

20. Carvalho AMP, Cataneo C, Galindo EMC, Malfará T. Autoconceito e imagem corporal em crianças obesas. Paidéia. 2005;15:131-9.

21. Malta DC, Goulart EMA, Costa MFFL. Estado nutricional e variáveis sócio-econômicas na repetência escolar: um estudo prospectivo em crianças da primeira série em Belo Horizonte, Brasil. Cad Saúde Pública. 1998;14:157-64.

22. Suñé FR, Dias-da-Costa JS, Olinto MTA, Pattussi MP. Prevalência e fatores associados para excesso de peso e obesidade em escolares de uma cidade no Sul do Brasil. Cad Saúde Pública. 2007;23:1361-71.

23. Silva GAP, Balaban G, Motta MEFA. Prevalência de excesso de peso e obesidade em crianças e adolescentes de diferentes condições socioeconômicas. Rev Bras Saude Mater Infantil. 2005;15:37-42.

24. Ronque ERV, Cyrino ES, Dórea VR, Helio Serassuelo Jr, Galdi EHG, Arruda M. Prevalência de excesso de peso e obesidade em escolares de alto nível socioeconômico em Londrina, Paraná, Brasil. Rev Nutr. 2005;18:709-17.

25. Fagundes ALN, Ribeiro DC, Naspitz L, Garbelini LEB, Vieira JKP, Silva AP, et al. Prevalência de excesso de peso e obesidade em escolares da região de Parelheiros do município de São Paulo. Rev Paul Pediatria. 2008;26:212-7.

26. Berkey CS, Rockett HRH, Gillman MW, Field AE, Colditz GA. Longitudinal study of skipping breakfast and weight change in adolescents. Int J Obes Relat Metab Disord. 2003;27(10):1258-66.

27. Sonneville KR, LaPelle N, Taveras EM, Gillman MW, Prosse LA. Economic and other barriers to adopting recommendations to prevent childhood obesity: results of a focus group study with parents. BMC Pediatr. 2009;9:1-7.

28. Triches RM, Giugliani ERJ. Obesidade, práticas alimentares e conhecimentos de nutrição em escolares. Rev Saúde Pública. 2005;39:541-7.

29. Costa CRB. A influência da atividade física escolar na prevalência da obesidade infantil como um indicador para a promoção de saúde e sua relação com aspectos familiares e socioeconômicos. (dissertação). (Franca - não publicada): Universidade de Franca; 2008. p. 65.

30. Pimenta APA, Palma A. Perfil epidemiológico da obesidade em crianças: relação entre televisão, atividade física e obesidade. Rev Bras Ciên Mov. Brasília. 2001;9:19-24.

31. Welk G. Physical Activity Assessments for Health-Related Research. Champaign: Human Kinetics; 2002. 